\title{
COMPLEX OPTICAL CHARACTERIZATION OF MESH IMPLANTS AND ENCAPSULATION AREA
}

\author{
VALERIY P. ZAKHAROV*, IVAN A. BRATCHENKO*, \\ VLADIMIR I. BELOKONEV ${ }^{\dagger}$, DMITRY V. KORNILIN* \\ and OLEG O. MYAKININ* \\ *Samara State Aerospace University \\ Samara 443086, Moskovskoe Shosse 34, Russia \\ †amara Medical University \\ Samara 443099, Polevaya Street 80, Russia \\ ثud_liche@mail.ru
}

Received 6 November 2012

Accepted 25 December 2012

Published 5 March 2013

\begin{abstract}
Complex investigation of mesh implants was performed involving laser confocal microscopy, backscattered probing and OCT imaging methods. The growth of endomysium and fat tissue with microcirculation vessels was observed in the mesh encapsulation region. Confocal microscopy analysis shows that such pathologies complications such as necrosis formation and microcavities were localized in the area near implant fibers with the size compatible with fiber diameter. And the number of such formations increase with the increase of the size, number and density of microdefects on the implant surface. Results of numerical simulations show that it is possible to control implant installation up to the depth to $4 \mathrm{~mm}$ with a help of backscattering probing. The applicability of OCT imaging for mesh implant control was demonstrated. Special two-stage OCT image noise-reduction algorithm, including empirical mode decomposition, was proposed for contrast increase and better abnormalities visualization by halving the signal-tonoise ratio. Joint usage of backscattered probing and OCT allows to accurately ascertain implant and surrounding tissue conditions, which reduces the risk of relapse probability.
\end{abstract}

Keywords: Encapsulation area; laser confocal microscopy; mathematical simulation; OCT image.

\section{Introduction}

The purpose of numerous investigations of various implants is to find an effective material and construction, which would provide a combination of high biomechanical characteristics with the efficiency of transplantation and the absence of post-operation after effects and relapses. ${ }^{1}$ Mesh implants are produced by spinning of artificial fibers of various chemical composition and thickness, using thin single or complex filaments. ${ }^{2}$ These implants are about several hundreds of micrometers thick, with the fiber diameter in the range of 50-200 $\mu \mathrm{m}$. Generally, the

This is an Open Access article published by World Scientific Publishing Company. It is distributed under the terms of the Creative Commons Attribution 3.0 (CC-BY) License. Further distribution of this work is permitted, provided the original work is properly cited. 
most important characteristics determining the choice of a particular implant are the absence of toxicity, compatibility with organism tissues and the possibility of fast and reliable sterilization. At the same time, the transplantation efficiency in many practically important cases depends on implant localization among different anatomical structures, stimulation of surrounding tissue regeneration and formation of encapsulation area.

The post-operative control of encapsulation area should be carried out for the reduction of relapse probability after the implantation. ${ }^{3}$ Implant installation may cause complications such as microhemorrhages, intoxication and even the appearance of the necrosis. Detailed investigation of encapsulation area should be performed for clear understanding of implant and tissues splicing. Taking account the common mesh implant fiber diameter these studies should to be held with optical precision.

For high-resolution three-dimensional monitoring it is necessary to ensure both resolution level of several microns and penetration depth of a few millimeters. This requirement may be satisfied by differential backscattering probing and optical coherent tomography (OCT). Differential backscattering can provide information about tissues optical properties and correspondingly about type of examined tissue, OCT technology allows nondestructive imaging of the tissue at a depth up to several millimeters with resolution about 5 microns. ${ }^{4}$ High frame rate, signal-to-noise ratio (SNR) and spatial resolution as well as the ability to image over a large field of view are all equally important factors for accurate visualization and analysis of encapsulation area. Taken in account that mesh implant as usual localized at a marginal depth for OCT, the scattered effects makes OCT images blurred, and thus OCT images have to be optimized to improve their quality.

Several techniques for reduction of coherent and incoherent noise in OCT images have been used, ${ }^{5}$ including cross-correlation image alignment followed by weighted averaging, ${ }^{6}$ compounding several B-scans acquired at neighboring positions in the sample, ${ }^{7}$ and elastic spatial registration of several OCT B-scans followed by averaging. ${ }^{8}$ But the best results may be achieved if the method of image reconstruction will be based on characteristic tissue structure changes of the encapsulation area during intergrowth of mesh prosthesis and surrounding tissue. Thus, it is very important to have true knowledge of tissue structures and common types of various complications in encapsulation area with highest possible resolution not less than $1 \mu \mathrm{m}$. Such resolution may be achieved using confocal laser microscopy ${ }^{9}$ which yields high-contrast images.

\section{Instrumentation}

\subsection{Laser confocal microscopy system}

The confocal laser microscopic system was developed based on an inverted Olympus 1X71 optical microscope with a Yokogawa1 CSU-X1 scanning head, which operates as a Nipkov disk. ${ }^{10}$ The laser source was an ALC-400 module with fiber acoustooptic control of radiation of solid-state DPSS lasers with wavelengths of 488 and $532 \mathrm{~nm}$. Images were recorded using an Andor iXONEM EMCCD camera.

Inverted microscope Olympus was equipped with eyepieces providing 20 -fold increase and the system with interchangeable lenses (magnification 20X and 40X). Was achromatic nonimmersive lenses from UIS MPlan series with a numerical aperture of 0.4-0.65 which guarantee samples field of view in a range of $0.275-0.55 \mathrm{~mm}$ ? The full width at halfmaximum (FWHM) resolution of the confocal laser microscopy system was 0.466 microns.

Computer control of laser sources, digital camera and filter drums made it possible to record up to 2000 optical slices per second. Special Andor iQ software allowed us to use effective way of noise reduction - averaging over several frames of scanned image.

\subsection{OCT system}

In this study, we present first application of spectral domain OCT for mesh implants monitoring (see Fig. 1). The system comprises of a broadband superluminescent laser diode $(840 \pm 25 \mathrm{~nm}$ wavelength range, $20 \mathrm{~mW}$ output power) at the source end, Michelson interferometer with 50/50 split ratio to the sample and reference arms and a spectrometer at the detector end. The spectrometer comprises of a diffraction grating (1200 grooves/ $\mathrm{mm}$ ) and a CCD line scan camera (2048 pixel resolution, $29.3 \mathrm{kHz}$ line rate). The interference signal from the sample and the reference arms of the Michelson interferometer is detected by the spectrometer and digitized by an image acquisition card 


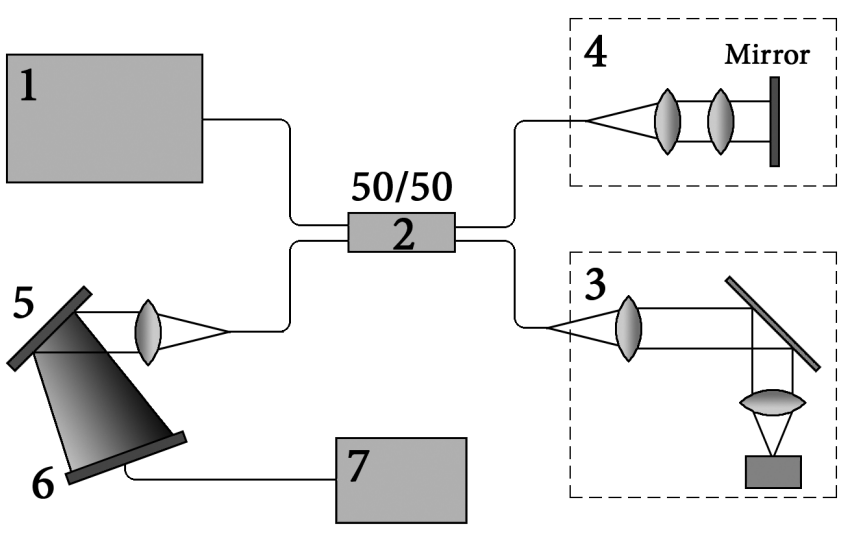

Fig. 1. Spectral domain OCT scheme: 1 - broadband laser source, 2 - 50/50 beamsplitter, 3 - sample arm, 4 - reference arm, 5 - spectrometer with grating, 6 - CCD camera, 7, 8 computer with IMAQ.

(NI-IMAQ PCI-1428). Depth profile (A-line) is obtained by converting the interference signal detected by the IMAQ into linear $k$-space.

\section{Laser Confocal Microscopy Study}

Experimental studies were performed on 16 white laboratory rats of both sexes, with a mass from $180-230$ g. All experiments were approved by Commission of Animals Ethical Treatment of Samara Medical University. Prolene Ethicon or Esfil standard propylene implants were implanted into the abdominal wall of the rats under ether anesthetic. The control group included four animals. The rats were observed for nine months after the implantation, after which they were killed with ether overdose. The abdominal wall with a polypropylene implant and adjacent tissue was extracted for microscopic studies. The extracted material was fixed in $10 \%$ neutral formalin. Serial sections were made (thickness up to $0.5 \mathrm{~mm}$ ), which were dyed with hematoxylin-eosin and picrofuchsin by Van Gieson method (Schiff reaction according to McManus).

There were no observed tissue changes in control group [see Fig. 2(a)]. All control group animals have strong muscle tissue in abdominal wall area. After implant installation (in test group animals) one can see a number of post-operative changes in the encapsulation area (examples on Figs. 2(b) and 2(c)). After nine months of Esfil implant transplantation, the mesh cells were surrounded by a strong connective tissue closely covering the polypropylene filaments for most observed animals from test group (four of six animals). The mature collagen fibers are located concentrically, and large rounded cells with large nuclei are directly adjacent to the surface of fibers. There are some areas of lymphocytic infiltration, which indicate microhemorrhages and necrosis formation.

Signs of autoimmune process for Prolene Ethicon implant were observed. In particular, the muscular tissue is edematous, there is a proliferation of endomysium and some fibers have myocytolysys phenomenon. Macrophages are present near atrophied muscular fibers. The form of muscular tissues is changed from polygonal to round. The irregular growth of collagen fibers (immature granulation tissue) is observed in the implant region around polypropylene filaments. There are a large number of adipose cells with thinned cytolemma and lots of microvasculature vessels [see Fig. 2(b)]. The cytoplasma is almost entirely occupied by vacuole in adipose cells. Hyperemia is observed in the microvascular.

The growth of endomysium and fat tissue (which contains many microcirculation vessels) is observed in the mesh encapsulation region for implants of both types. Some regions between the connecting

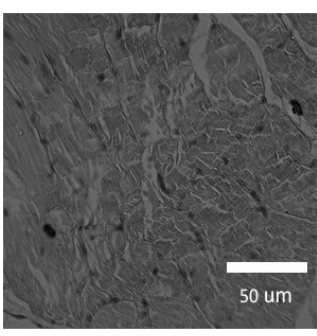

(a)

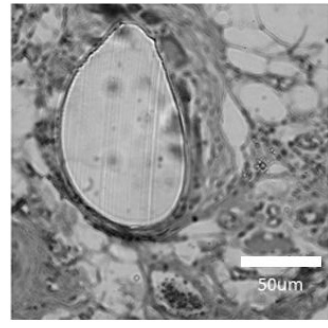

(b)

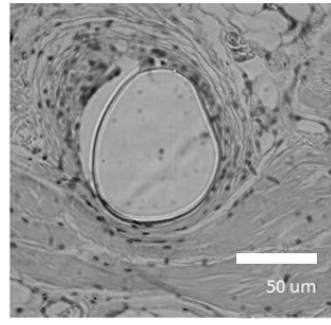

(c)

Fig. 2. Confocal microscopy images: (a) normal muscle tissue from control group animal and pathologies in encapsulation area, (b) the substitution of normal tissue by adipose cells, and (c) voids formation. 
tissue and fibers contain cavities [see Fig. 2(c)]. The formation of voids on the tissue-implant fiber border poses a threat to the integrity of the entire encapsulation area. In this situation, the implant motions in tissue leads to its mechanical damage, cutting and microtrauma formation. The probability of such microtrauma formation increases with the increase of the size, number and density of microdefects on the implant surface. These defects not only expand the void size and change micromechanical properties of implant but also prevent normal encapsulation area formation and may cause more significant damage of the tissue such as haemorrhage. The timely diagnosis of such pathologies presence can help to avoid serious injuries of tissues in encapsulation area and will decrease relapse probability after transplantation.

\section{Differential Backscattering of Encapsulation Area}

The microscopic studies show that the main pathological changes during the recovery of encapsulation area are related with normal tissue substitution by adipose cells and necrotic tissue formation near the implant fibers. Such pathological alterations are accompanied by loss of normal tissue elasticity, which leads to mechanical damage and possible shift of the implant. The precise diagnostics of the above-discussed pathologies in their early stage makes it possible to start timely treating, which reduce the risk of relapse. Note that, in view of the wide wound field, monitoring of the post-operation wound process and detection of possible pathology development can be performed based on the differential analysis of scattered light and detection of inhomogeneity related to the presence of necrotic tissue and/or dystrophic changes in the tissue adjacent to the region occupied by implant fibers.

The mathematical model of multi-layer biological medium ${ }^{3}$ was used for estimation of wound process monitoring possibility with a help of optical backscattering. The distribution of scattered light intensity was determined by Monte Carlo technique $^{11}$; the numerical values of the optical absorption and scattering coefficients were chosen based on the analysis of the experimental and model data $^{11,12}$ with subsequent certification in numerical model experiments. The form, topology and sizes of mesh implants were chosen based on the samples used in medical practice. ${ }^{3}$ The RMS error does not exceed $6.2 \%$ for all simulated numeric experiments. The topology model is represented in Fig. 2, where $D$ - diameter of implant fiber and $d$ - the thickness of necrosis layer around the implant surface. Mesh implant structure is represented in Fig. 2(b), and frames reconstruction of three-dimensional confocal microscopy images is shown in Fig. 3(c).

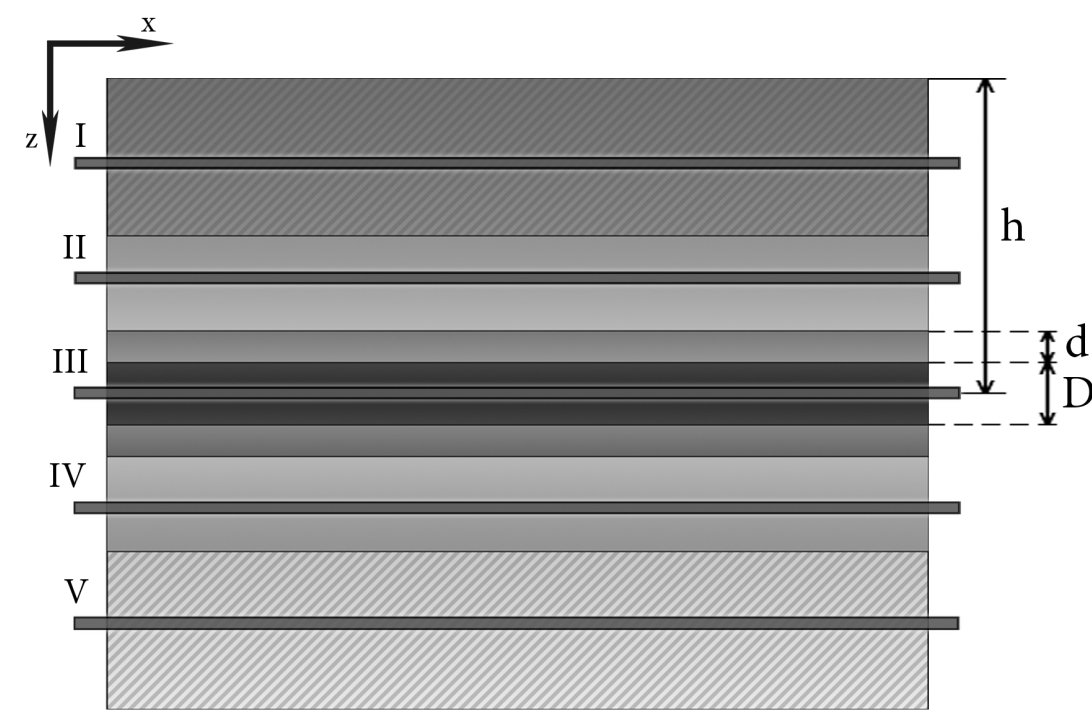

(a)

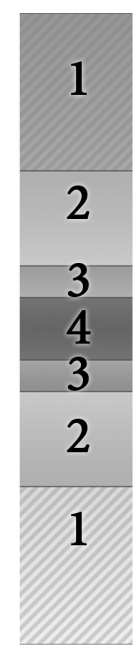

Fig. 3. Topology of tissue with mesh implant: (a) normal tissue (1) and implant fiber (4) surrounded by necrosis layer (3) in encapsulation area (2), (b) mesh implant cross-section on the depth $\mathrm{h}$, and (c) confocal microscopy frames for 3D image reconstruction. 


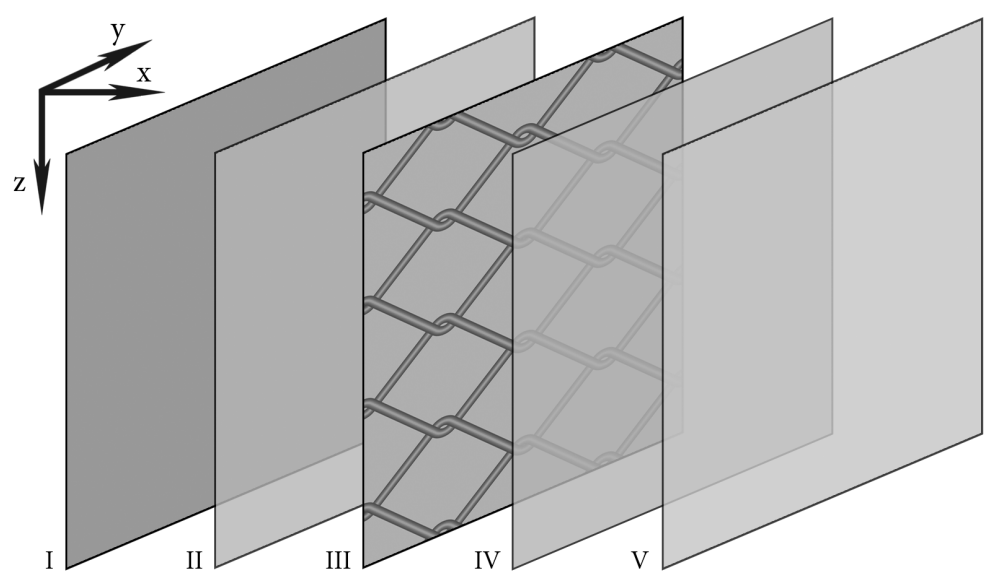

(c)

Fig. 3. (Continued)

Actually, an implant is a distributed scatterer with sharp optical boundaries, located at a certain depth in a muscular tissue. Hence, the light scattering coefficient of the implant is almost completely determined by the Fresnel coefficients, and the possibility of its detection is determined by the absorption and scattering coefficients of the tissue.

Figure 4 shows the maximum depth at which the implant's grid may be resolved by Rayleigh criteria. Curves 1 (scattering coefficients of $60 \mathrm{~mm}^{-1}$ and the absorption of $0.34 \mathrm{~mm}^{-1}$ ) and 2 (the scattering coefficients of $45 \mathrm{~mm}^{-1}$ and the absorption of $0.44 \mathrm{~mm}^{-1}$ ) in Fig. 4 is actually "cuts" the area of

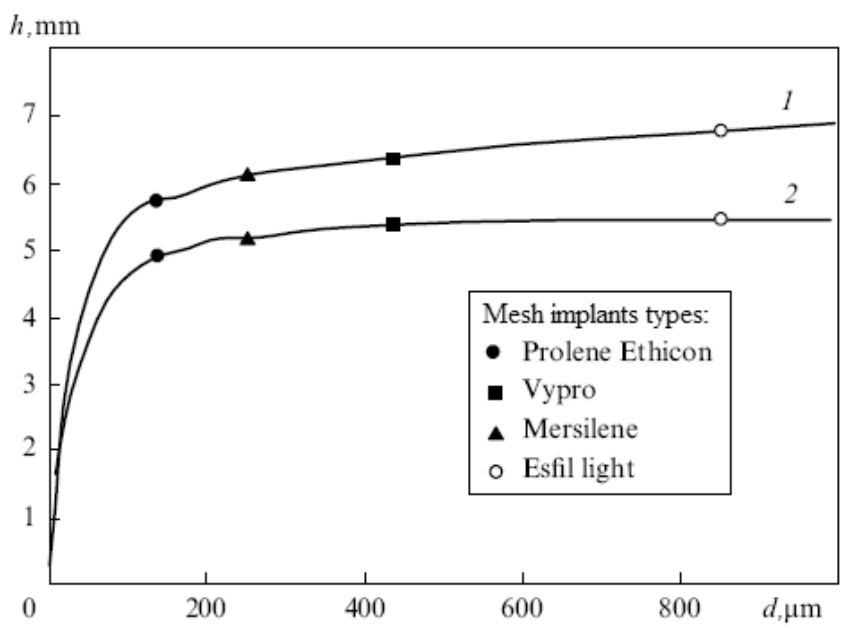

Fig. 4. Dependence of the limiting visualization depth $(h)$ of mesh implant structure from the effective cell diameter $(d)$ and medium characteristics. Scattering and absorption coefficients are (1) 60 and $0.34 \mathrm{~mm}^{-1}$ and (2) 45 and $0.44 \mathrm{~mm}^{-1}$, respectively. visualization corresponding to normal muscle tissue absorption and scattering coefficients dispersion. Saturation region starting with the value of $h \sim$ $5 \mathrm{~mm}$ is due to the medium absorption.

The threshold depth when absorption becomes dominant is about $5 \mathrm{~mm}$ and according to the Rayleigh criterion most mesh implants can be resolved at a depth which exceeds $4 \mathrm{~mm}$. According to the microscopic studies, encapsulation process may be significantly affected by the necrotic formations whose absorption spectra range corresponds to $440-600 \mathrm{~nm}$. It is clear that the possibility to detect necrotic structure will depend on its size and the depth of mesh implant. Then, for characterization of possibility to detect such pathology it is convenient to introduce the effective diameter of necrotic formation, which is defined as the ratio of the average diameter of resolved necrotic formation $d$ to the diameter of mesh implant fiber $D$.

Figure 5 shows the dependence of the minimum effective diameter on its location depth for the model of Prolene Ethicon implant. One can see that this dependence is linear up to a depth of $1.4 \mathrm{~mm}$ (the minimum visible effective formation diameter does not exceed the implant fiber diameter), and, starting from depth $h \sim 1.5 \mathrm{~mm}$, it becomes highly nonlinear due to the enhanced role of light absorption and diffusion. As usual, necrotic formation size is comparable with the fiber diameter, so as one may see from Fig. 5 that the backscattering light analysis has substantial limitations in application of encapsulation area control.

The process of implant and tissue splicing is accompanied by changes in the encapsulation area 


\section{P. Zakharov et al.}

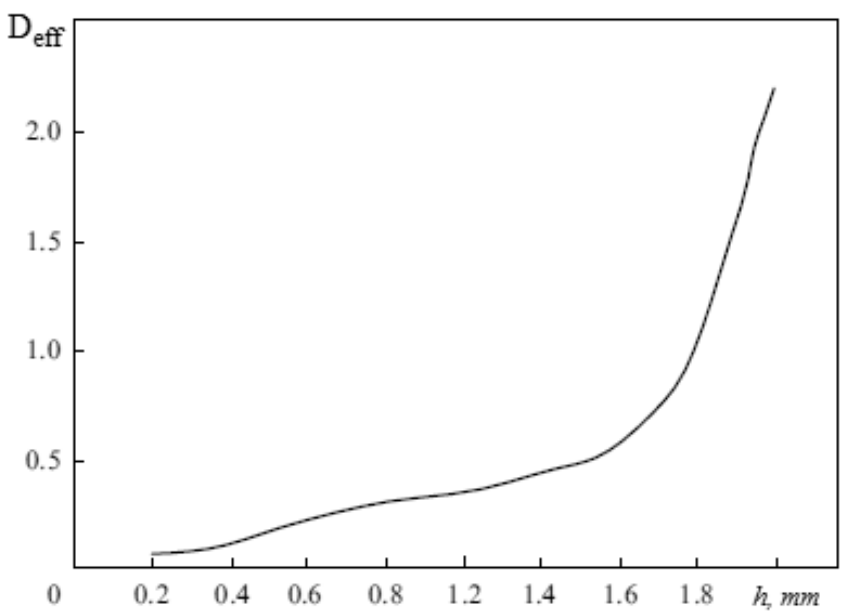

Fig. 5. Dependence of the minimum resolved effective diameter $(D)$ of necrotic formations on the implant fiber surface, normalized to the mesh fiber diameter, on the mesh location $\operatorname{depth}(h)$.

optical characteristics. On the first day of implant installation, surrounding tissue has a large number of dead cells, which is characterized by a high absorption in the encapsulation area; this fact does not allow the visualization of fiber prosthesis. While as the time passes, there is tissue repair and replacement of dead cells with new ones. This leads to changes in medium optical properties: strong absorption (in the case of necrotic tissue) is replaced by a much smaller absorption of normal muscle tissue. A reflection of this fact is the change in the optical thickness of encapsulation area. Consequently, optical control of encapsulation area after transplant surgery gives an indication of how well the tissue is healing after surgery.

\section{OCT in Encapsulation Area Analysis}

Backscattering probe gives two-dimensional results, and its resolution depends on the depth of the implant installation. The main aim of OCT usage was to ensure three-dimensional control of encapsulation area with higher resolution. Experimental tests were performed on the model mediums which were prepared on the basis of mesh implants coated with biological tissue layers.

One of the frames of direct acquisition of OCT data, which involves scanning a beam along two directions, is presented on Fig. 6(a). Test implant with mesh cell size $1 \mathrm{~mm}$ was formed by complex multi-filament polytetrafluoroethylene fibers with

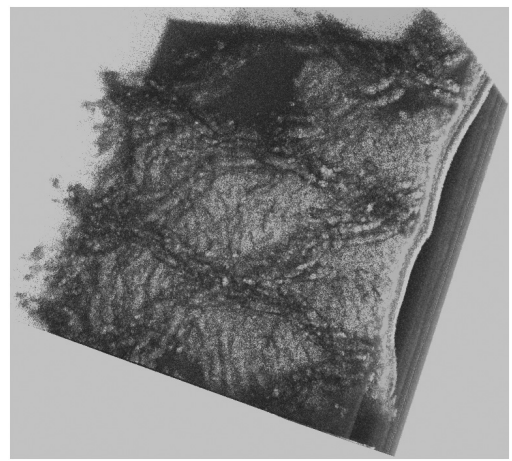

(a)

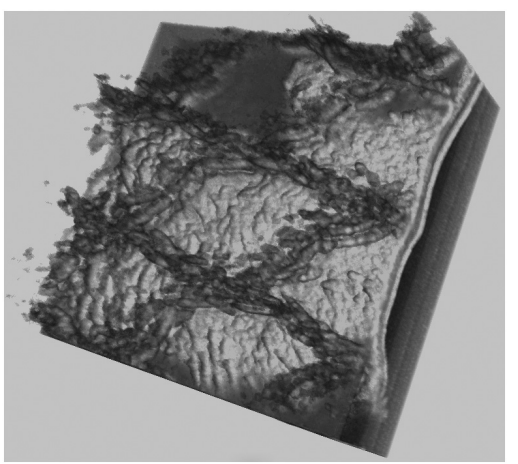

(b)

Fig. 6. OCT image of implant under the layer of mouse skin: (a) raw and (b) EMD-filtered.

diameter $20 \mu \mathrm{m}$ and tissue layer thinness was $1.7 \mathrm{~mm}$.

Recorded images have low SNR and likewise all coherent imaging systems. OCT suffers from speckle noise. We assume that the imaged structures either are static or undergo a slow periodic motion that follows the breath. We also assume that additive noise contributing to the image at each space-time location follows a zero-mean random distribution and is uncorrelated with the signal.

We used two-stage OCT image denoising algorithm for an accurate diagnosis of mesh implant and encapsulation area: simple pre-filtering (interframe averaging and linear smoothing) on the first stage and empirical mode decomposition (EMD-filtering) on the second stage. The aim of the first stage is to remove ultrahigh frequency modes from the raw data which as usual does not include any useful information about the investigated object. The second stage must reduce remaining noise spikes at high frequencies without affecting the fine details of the desired signal. 
Raw three-dimensional digital OCT signal can be represented as a set of depthward cross-section B-scan images. As OCT longitudinal resolution is about $5 \mu \mathrm{m}$ each neighboring images cannot significantly differ in structure details. Such first stage of denoising algorithm may be realized as interframe averaging of $K$ adjacent $\mathrm{C}$-scans with subsequent $3 \times 3$ spatial average linear smoothing (convolution) of each frame. The choice of parameter $K$ is individual and depends on images dynamics.

The second stage EMD-filtering is based on onedimensional algorithm of spatial frequency analysis ${ }^{13}$ with sequential filtering of high frequencies. EMD-procedure is applied for each row (or each column) of the C-scan. As a result, the original signal is divided into a sum of $N$ quasi-orthogonal quasi-periodic intrinsic mode functions (IMFs) and residual.

Authors in Ref. 14 proposed to reject first modes for further noise reduction. But such procedure leads to the loss of small-size image details. Taking into account that the signal amplitude is much larger than the noise amplitude we perform thresholding over the first two IMFs, which replace by zero all their spatial half-periods with amplitude less than some pre-assigned threshold $\mathrm{T}$. The results of EMD-filtering is shown on Fig. 6(b). The figure shows that the noise was reduced and the small details were saved.

We estimate the values of SNR as Signal variance value divided by Noise variance value for all C-scans of 3D OCT image/ Method for estimation of Noise variance has been described in Ref. 15 . Noise reduction results are summarized in Table 1. Comparison of minimum and mean SNR values for noise reduction algorithm stages was performed.

The first stage of noise removal leads to slight increase of SNR (about 17\%) while the second stage greatly increases SNR up to two times and higher. As one can see, the lower boundary of SNR also increases. This allows drawing a conclusion of the necessity of noise reduction of OCT images for

Table 1. SNR characterizations for 3D OCT image of implant on Fig. 6.

\begin{tabular}{lccc}
\hline & & & After \\
& Original & After 1st stage & 2nd stage (EMD) \\
\hline Mean SNR & 0.748 & 0.877 & 1.863 \\
Min SNR & 0.023 & 0.219 & 0.680 \\
\hline
\end{tabular}

correct mesh implant control. As it may be seen from Fig. 6 it will enable the possibility of in vivo OCT monitoring of implant and surrounding tissues. The decrease of noise level helps to see even small details, register and localize several micronssize tissue anomalies and start up appropriate treatment on the early stages of disease course.

\section{Conclusions}

In summary, with a help of laser confocal microscopy it was proved that the main pathologies complications for mesh implants are related with the substitution of normal tissue by adipose cells and void formation, which leads to necrotic microtrauma in encapsulation area near the implant fibers. In order to reduce relapse probability it is necessary to perform post-operational monitoring of implants and encapsulation area. Monte Carlo simulations show the possibility of implant detection up to the depth of $4 \mathrm{~mm}$, but encapsulation area details may be observed only up to the depth of $1.8 \mathrm{~mm}$.

We have demonstrated the applicability of backscattering probing for two-dimensional and OCT imaging for three-dimensional mesh implant control with the ability of accurate pathologies localization. As OCT must operate on threshold depth it was proposed and validated the two-stage noise-reduction algorithm, including interframe averaging, linear smoothing and empirical mode decomposition.

\section{Acknowledgments}

This research was supported by the Federal Target Program "Scientific and scientific-pedagogic staff of innovative Russia in 2007-2013".

\section{References}

1. A. G. Kirpichev, N. A. Surkov, Use of Prolen Mesh in the Case of Plastic of the Front Abdominal Wall, Media Sfera, Moscow (2001).

2. S. C. William, W. K. Kent, "Intra-abdominal placement of antimicrobial-impregnated mesh is associated with noninfectious fever," Surg. Innov. 12, 63 (2005).

3. V. P. Zakharov, V. I. Belokonev, I. A. Bratchenko, P. E. Timchenko, Yu. V. Ponomareva, A. V. Vavilov, L. T. Volova, "Application of confocal laser microscopy 
for monitoring mesh implants in herniology," Quantum Electron. 41(4), 318-323 (2011).

4. M. Wojtkowski, "High-speed optical coherence tomography: Basics and applications," Appl. Opt. 16 (49), 30-61 (2010).

5. J. Schmitt, S. Xiang, K. Yung, "Speckle in optical coherence tomography," J. Biomed. Opt. 4(95), (1999).

6. B. Sander, M. Larsen, L. Thrane, J. Hougaard, T. M. Jørgensen, "Enhanced optical coherence tomography imaging by multiple scan averaging," British. J. Ophthamol. 89, 207 (2005).

7. D. P. Popescu, M. D. Hewko, M. G. Sowa, "In vitro assessment of optical properties of blood by applying the extended Huygens-fresnel principle to timedomain optical coherence tomography signal at 1300 nm," Opt. Commun. 269, 247 (2007).

8. T. M. Jørgensen, J. Thomadsen, U. Christensen, W. Soliman, B. Sander, "Enhancing the signal-tonoise ratio in ophthalmic optical coherence tomography by image registration - Method and clinical examples," J. Biomed. Opt. 12, 041208 (2007).

9. M. Rajadhyaksha, "In vivo confocal scanning laser microscopy of human skin II: Advances in instrumentation and comparision to histology," J. Invest. Dermatol. 113, 293 (1999).
10. M. D. Egger, M. Petran, "New reflected-light microscope for viewing unstained brain and ganglion cells," Science 157, 305-307 (1967).

11. V. P. Zakharov, A. R. Sindyaeva, "Multi-parametric function of differential backscattering in $3 \mathrm{~d}$ biological media with heterogeneities," Laser Phys. 19, 1361-1365 (2007).

12. V. V. Tuchin, Tissue Optics: Light Scattering Methods and Instruments for Medical Diagnosis, 2nd edn., SPIE Press, Bellingham (2007).

13. N. E. Huang, Z. Shen, S. R. Long, M. C. Wu, H. H. Shih, Q. Zheng, N.-C. Yen, C. C. Tung, H. H. Liu, "The empirical mode decomposition and the Hilbert spectrum for nonlinear and non-stationary time series analysis," Proc. Roy. Soc. London A 454, 903-995 (1998).

14. M. B. Bernini, A. Federico, G. H. Kaufmann, "Normalization of fringe patterns using the bidimensional empirical mode decomposition and the Hilbert transform," Appl. Opt. 48, 6862-6869 (2009).

15. D. Donoho, I. Johnstone, "Ideal spatial adaptation via wavelet shrinkage," Biometrika 81, 425-455 (1994). 